\title{
Diffractive imaging of highly focused X-ray
} fields

\author{
H. M. QUINEY ${ }^{1}$, A. G. PEELE ${ }^{2}$, Z. CAl ${ }^{3}$, D. PATERSON ${ }^{3}$ AND K. A. NUGENT ${ }^{1 *}$ \\ ${ }^{1}$ School of Physics, The University of Melbourne, Victoria 3010, Australia \\ ${ }^{2}$ Department of Physics, La Trobe University, Bundoora, Victoria 3086, Australia \\ ${ }^{3}$ Advanced Photon Source, Argonne National Laboratory, Argonne, Illinois 60439, USA \\ *e-mail:k.nugent@physics.unimelb.edu.au
}

$\mathbf{T}$ The rapid development of new sources of coherent X-rays, such as third-generation synchrotrons, high-harmonicgeneration lasers ${ }^{1}$ and $\mathrm{X}$-ray free-electron lasers ${ }^{2}$, has led to the emergence of the new field of X-ray coherent science. The extension of coherent methods to the $\mathrm{X}$-ray regime makes possible methods such as coherent diffraction, X-ray photoncorrelation spectroscopy, speckle interferometry and ultrafast probing at atomic resolution and femtosecond timescales. Despite rapid improvements in the resolution that conventional $\mathrm{X}$-ray optics can achieve, new methods for manipulating X-rays are required to push this to the atomic scale ${ }^{3}$. Here we demonstrate a coherent imaging technique that enables us to image the complex field at the focus of an X-ray zone plate without the need for conventional X-ray lenses. There are no fundamental limits on the resolution of this lensless imaging technique other than the wavelength of the X-rays themselves. The ability to characterize the beam with one measurement makes the method ideally suited to characterizing the fields generated by pulsed coherent $\mathrm{X}$-ray sources.

In ref. 4, it was shown that the Fraunhofer diffraction pattern from an isolated non-periodic object was sufficient to fully reconstruct the diffracting object. Methods, based on modifications ${ }^{5}$ of the Gerchberg-Saxton ${ }^{6}$ algorithm developed for electron microscopy, have been used to demonstrate the reliable reconstruction of objects from the coherent scattered X-ray data ${ }^{7}$. The reconstruction method relies on the knowledge that the diffracting object has a finite extent. Methods now exist for the extent of the object to be refined during the course of the iteration ${ }^{8}$, but all methods fail for objects that violate what is known as the oversampling requirement ${ }^{9}$. This means that the diffraction data must be sampled at least densely enough to enable the reconstruction of the autocorrelation function of the scattering object.

When X-rays pass through a focusing device, the focal distribution obeys a Fourier transform relationship with the exit pupil of the optical system. Similarly, if the X-ray distribution is measured at a sufficiently large distance from the focus then, provided that a spherical phase curvature from the lens is retained (see the Methods section), the detected field has a Fourier relationship with the field at the focus. However, the exit pupil

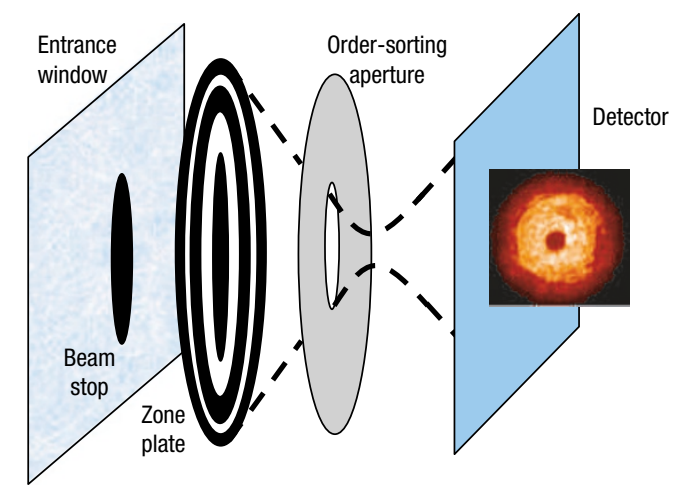

Figure 1 Schematic of the experimental arrangement. A logarithmic plot of the measured intensity for the 2-ID-D experiment is also shown.

of the focusing system is, of necessity, finite and so the focal distribution has, at least in principle, infinite extent. It is certainly not possible to impose a well defined spatial support for the focal distribution, as would be required for the application of the previously developed approaches to coherent diffractive imaging. However, it is known that the field is generated from an optical system for which the shape of the exit pupil is well defined. In this letter, we use this knowledge as the a priori information with which to constrain our solution. That is, we use known support information in a plane other than that of the object to be recovered. We note that related methods have been used involving support information from other planes for the characterization of optical systems ${ }^{10}$, such as in the Hubble telescope $\mathrm{e}^{11}$. The complex Wigner deconvolution methods for X-ray imaging have also been used $^{12}$ and data were obtained that would have allowed the pupil of the system to be reconstructed, although this step was not actually taken.

The Gerchberg-Saxton algorithm iteratively finds a solution consistent with measured data and known constraints. This idea need not be restricted to the Fraunhofer plane and it 

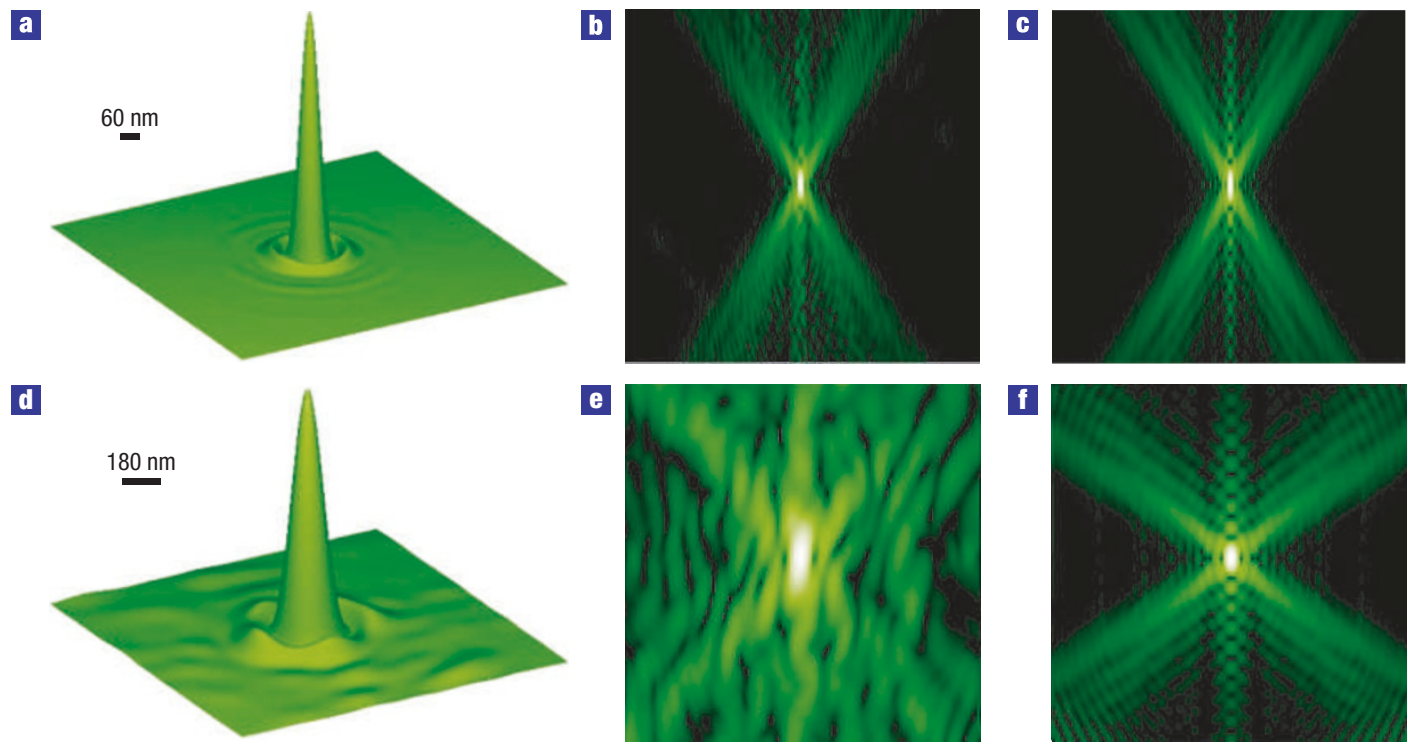

Figure 2 Plots of the field reconstructed around the focal point of each of the zone plates. a, Isometric plot of the intensity at the focal plane of the 2-ID-B zone plate. b, A meridional slice through the reconstruction of the three-dimensional intensity distribution. c, A calculation of the data shown in $\mathbf{b}$ using the nominal zone-plate parameters and a central stop. The optical axis of the system runs vertically through $\mathbf{b}-\mathbf{c}$ and $\mathbf{e}-\mathbf{f}$. $\mathbf{d}-\mathbf{f}$, As for $\mathbf{a}-\mathbf{c}$ but for the 2-ID-D zone plate. Note that these data sets are on different spatial scales and so the scale bar shown in a is $60 \mathrm{~nm}$ in length and the scale bar shown in $\mathbf{d}$ is $180 \mathrm{~nm}$ in length.

has been shown that the solution is unique in the case of Fresnel diffraction ${ }^{13}$. It might be expected that iterative phase recovery should therefore be more reliable when applied to Fresnel diffraction, and this has been demonstrated using theoretical and computational arguments ${ }^{14}$.

Owing to the very small size of the focus, which may be as small as ${ }^{15} 15 \mathrm{~nm}$, it is not practical to use direct methods such as a knife-edge scan across the focus. Instead, here we apply the indirect methods of coherent diffractive imaging to the intensity distribution, measured a large distance downstream of the focus. As a support constraint on our solution, we require that the field be fully contained within the known pupil of the zone plate, and this is enforced by propagating the focal distribution to both the pupil plane to enforce the support constraint, and to the measurement data to impose the measured intensity distribution.

The experimental arrangement is very simple and is shown schematically in Fig. 1. In our experiment, we obtained two sets of data. The first was acquired at the 2-ID-B intermediate-energy undulator beamline at the Advanced Photon Source, Argonne National Laboratory. An X-ray energy of $1.8 \mathrm{keV}$ was used. This beamline uses a spherical grating monochromator to coherently illuminate the zone plate, which had an outermost zone width of $50 \mathrm{~nm}$ and a diameter of $80 \mu \mathrm{m}$. A $28-\mu \mathrm{m}$-diameter central stop was placed on the exit window of the beamline to remove the undiffracted beam from the focal region. A $20-\mu \mathrm{m}$-diameter order sorting aperture (OSA) was used to remove higher-order foci. The intensity of the defocused beam was directly detected using a back-side-illuminated, liquid-nitrogen-cooled Princeton Instruments charge-coupled device (CCD) camera with $1,024 \times$ 1,024 pixels, each $24 \mu \mathrm{m} \times 24 \mu \mathrm{m}$, placed $27 \mathrm{~cm}$ downstream of the focal spot. The data obtained looked very similar to those that would be created by a perfect zone plate. In our second data set, a high-coherence ${ }^{16} 8 \mathrm{keV} \mathrm{X}$-ray beam conditioned by slits, mirrors and a double-crystal monochromator from the undulator beamline 2-ID-D at the Advanced Photon Source was used. The zone-plate system consisted of two zone plates each with 400 zones, $160 \mu \mathrm{m}$ in diameter with an outermost zone width of $100 \mathrm{~nm}$. A 40- $\mu \mathrm{m}$ diameter central stop placed on the entrance window and a $30-\mu \mathrm{m}-$ diameter OSA were used. Images were recorded using a Mar-USA fibreoptic-coupled CCD camera with 2,048 $\times 2,048$ pixels, each $80 \mu \mathrm{m} \times 80 \mu \mathrm{m}$. The detector was placed $2.56 \mathrm{~m}$ downstream of the zone plate focus. The data for 2-ID-D are shown in Fig. 1. It can be seen that the defocused spot contains a large number of complex structures and is far from ideal.

The presence of the OSA largely eliminates the contribution of all orders other than the first; the OSA acts as a spatial filter on the recovered data and so removes detailed information about the zone structure of the zone plate. The sampling at the detector follows the usual rules for coherent diffraction imaging: the realspace resolution is determined by the highest angle detected, and the real-space field of view is determined by the sampling rate (that is, the CCD pixel size). The first-order focus reconstructed here falls rapidly to negligible levels over the field of view and we collect data out to angles well-beyond the maximum angle into which light is scattered. A reconstruction of the complete zone plate would, at least in principle, be possible but would require removal of the OSA.

The light from a third-generation synchrotron is not fully coherent and, in the 2-ID-D data here, the zone-plate aperture is not fully coherently illuminated. The iterative reconstruction algorithms assume a coherent wavefield. To understand the consequences of this, we simulated partially coherent illumination by blurring a simulated coherent experiment, so as to mimic the effects of a finite but incoherent synchrotron source. We then applied the coherent reconstruction techniques to these data. We found that the partial coherence had no effect on the reconstruction provided that the blurring due to partial coherence did not extend over more than two pixels at the detector. Within this criterion, the data are largely indistinguishable from a coherent data set and so, therefore, is the reconstruction. That is, for data that has high, but not full, coherence, the method reconstructs the point-spread function of the optic; it does not recover the broader partially coherent focus. In the 2-ID-D data, the blurring due to partial 


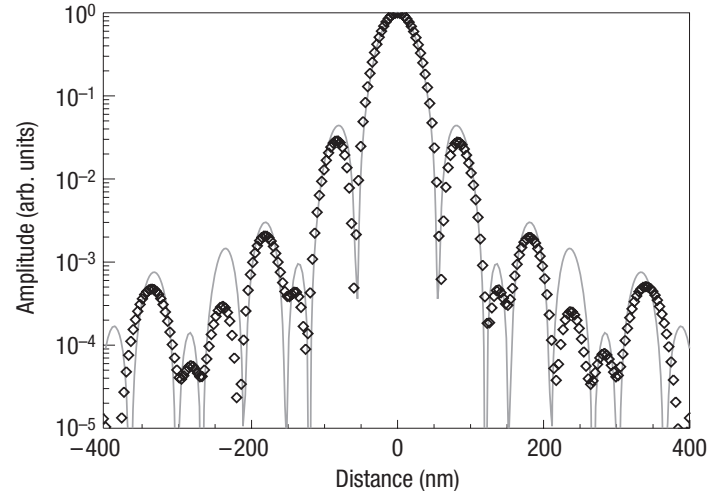

Figure 3 Logarithmic plot of the intensity of the reconstructed wavefield along one axis of the focal plane for the 2-ID-B data. The diamonds are from measurement and the line from theory. The reconstructions from different random starts fall within the plotted data points. The resolution by the Rayleigh criterion, which is the distance from the peak to the first minimum, is found to be $63 \pm 2 \mathrm{~nm}$. Note that the details in the sidelobes are accurately reconstructed.

coherence occurs over less than one pixel and so the effects are not seen in that data set. At the same time, the field of view is large enough that the reconstructed signal falls to negligible levels at its edges. Therefore, the partially coherent illumination has not measurably degraded the resulting image.

The data were reconstructed by iterating between the zoneplate pupil plane, focal plane and the measurement plane using a Fresnel diffraction algorithm (see the Methods section). A random phase was added to the measured amplitudes to begin the iterative scheme. The algorithm was found to converge smoothly, reliably and monotonically to the solution. The data sets were found to converge on very similar solutions, irrespective of the starting guess, and the differences between solutions were used to estimate the uncertainty in the reconstruction. To test the reliability of the reconstruction, different supports were used at the zoneplate plane. We found that a support that was known to be too small produced an iteration that failed to converge. A larger support produced a slower convergence and, with a support that was matched to the known pupil of the zone plate, convergence was obtained in 400-1,000 iterations. In both experiments the beamstop could be moved independently of the zone plate and so there is some uncertainty in their relative alignment. Accordingly, the 2-ID-B data used only the diameter of the zone plate as the support constraint. To improve convergence, the 2-ID-D data used an annular aperture incorporating a central stop as its support. A 10 - $\mu \mathrm{m}$-diameter stop was imposed to allow for errors in alignment. The resulting converged iteration showed a central stop with the correct diameter of $30 \mu \mathrm{m}$.

The reconstructed intensity distribution in the focus is shown in Fig. 2, where the two-dimensional focused intensity is shown as well as a reconstruction of a slice through the three-dimensional intensity distribution. This latter reconstruction requires both the phase and amplitude to be recovered and is compared with the calculated distribution based on an ideal zone plate with the nominal parameters. The agreement is excellent in the case of the 2-ID-B data, and the focus is clearly rather poorer than the ideal case in the 2-ID-D data.

The reconstruction for 2-ID-B data is almost identical to that for a theoretically ideal zone plate, and shows a focus that would produce a Rayleigh imaging resolution of $(63 \pm 2) \mathrm{nm}$, compared with an expectation of $61 \mathrm{~nm}$ (see Fig. 3). The reconstruction of the zone-plate pupil indicates that the slightly poorer resolution arises from non-uniform illumination of the zone plate, not phase errors in the focusing field. Figure 3 also shows agreement between theory and measurement of the detailed structure of the reconstructed focal wavefield, including the pattern of secondary peak values arising from the annular aperture of the optic, a feature that we emphasize was not built into the support information.

In the 2-ID-D case, some irregular structures around the intense focal region were found, as would be anticipated from an imperfect zone plate. In this case, the Rayleigh resolution of the zone plate is found to be $(180 \pm 8) \mathrm{nm}$. The main source of uncertainty is the inhomogeneity in the reconstructed focal spot. This compares to a measured limit to the resolution of over $150 \mathrm{~nm}$, measured using the fluorescence of a chromium edge ${ }^{17}$, and an ideal resolution of $122 \mathrm{~nm}$. The reconstructed phase variation across the optic is relatively small and is unlikely to broaden the focal spot greatly. We attribute the non-uniformity to phase errors in the beryllium entrance window, and to inaccuracies in the alignment of the two zone plates. This latter contribution is supported by a small, but significant, asymmetry in the focal spot. However, it was found that the transmission through the zone plate is very strongly peaked towards the centre of the optic, implying that the outer zones will be contributing relatively little to the focus. This deduction is confirmed by the strong non-uniformities in the measured data (Fig. 1). It is therefore the limitation on the effective pupil size that primarily degrades the focus.

This application of coherent diffractive imaging will find use in the immediate and complete characterization of pulses in the free-electron lasers currently being developed around the world. It will also enable the reliable characterization of high-resolution optical systems.

\section{METHODS}

We reconstruct the complex X-ray optical field using an algorithm adapted from the methods used in coherent diffractive imaging and in this section we describe the algorithm used.

The propagation in the $z$-direction of a planar wavefield $\psi\left(\boldsymbol{\rho}_{i}, z_{i}\right)$ from a plane at $z_{i}$ to $\psi\left(\boldsymbol{\rho}_{j}, z_{j}\right)$ at a parallel plane at $z_{j}$ is given in the paraxial Fresnel free-space approximation by

$$
\begin{aligned}
\psi\left(\boldsymbol{\rho}_{j}, z_{j}\right)= & -\frac{i}{\lambda z_{i j}} \exp \left(\frac{2 \pi i z_{i j}}{\lambda}\right) \exp \left(\frac{i \pi \boldsymbol{\rho}_{j}^{2}}{\lambda z_{i j}}\right) \\
& \times \iint \psi\left(\boldsymbol{\rho}_{i}, z_{i}\right) \exp \left(\frac{i \pi \boldsymbol{\rho}_{i}^{2}}{\lambda z_{i j}}\right) \exp \left(-\frac{2 \pi i \boldsymbol{\rho}_{i} \cdot \boldsymbol{\rho}_{j}}{\lambda z_{i j}}\right) \mathrm{d} \boldsymbol{\rho}_{i},
\end{aligned}
$$

where $\boldsymbol{\rho}_{i}$ defines the position in plane $z_{i}, \lambda$ is the wavelength of the illumination and $z_{i j}=z_{j}-z_{i}$. We may express equation (1) in a form that more accurately reflects how this is achieved in practice by writing

$$
\begin{aligned}
\psi\left(\boldsymbol{\rho}_{j}, z_{j}\right) & =-\frac{i}{\lambda z_{i j}} \exp \left(\frac{2 \pi i z_{i j}}{\lambda}\right) \exp \left(\frac{i \pi \boldsymbol{\rho}_{j}^{2}}{\lambda z_{i j}}\right) \hat{F}\left[\exp \left(\frac{i \pi \boldsymbol{\rho}_{i}^{2}}{\lambda z_{i j}}\right) \psi\left(\boldsymbol{\rho}_{i}, z_{i}\right)\right] \\
& =A\left(\boldsymbol{\rho}_{j}, z_{i j}\right) \hat{F}\left[B\left(\boldsymbol{\rho}_{i}, z_{i j}\right) \psi\left(\boldsymbol{\rho}_{i}, z_{i}\right)\right],
\end{aligned}
$$

where $\hat{F}$ denotes the Fourier transform operator, which is defined implicitly by equation (1). Propagation between any two planes according to equation (1) simply involves two simple multiplicative functions, $A\left(\boldsymbol{\rho}_{j}, z_{i j}\right)$ and $B\left(\boldsymbol{\rho}_{i}, z_{i j}\right)$, and one Fourier transformation.

We define the plane of the zone plate to be at $z_{1}$, the focal plane to be at $z_{2}$ and the detector at $z_{3}$, and the iterative determination of the wavefield follows the propagation cycle $z_{1} \rightarrow z_{2} \rightarrow z_{3} \rightarrow z_{2} \rightarrow z_{1}$. Numerical control of the 
algorithm is maintained by the substitutions

$$
\begin{gathered}
\psi\left(\boldsymbol{\rho}_{1}, z_{1}\right)=P\left(\boldsymbol{\rho}_{1}\right) \exp \left(-\frac{i \pi \boldsymbol{\rho}_{1}^{2}}{\lambda f}\right) \\
\psi\left(\boldsymbol{\rho}_{3}, z_{3}\right)=Q\left(\boldsymbol{\rho}_{3}\right) \exp \left(+\frac{i \pi \boldsymbol{\rho}_{3}^{2}}{\lambda z_{23}}\right),
\end{gathered}
$$

where $f=z_{12}$ is the first-order focal length of the zone plate. The combination of optical components consisting of the zone plate, OSA and beamstop have the effect that $P\left(\boldsymbol{\rho}_{1}\right)$ may be regarded as the complex pupil function of a thin lens of focal length $f$. This pupil function carries all information about imperfections and aberrations of the lens, and has a finite spatial extent, its support, which is imposed as a constraint. The most rapidly oscillating part of the imaged wavefield at $z_{1}$ is then handled analytically, with the result that the propagation $z_{1} \rightarrow z_{2}$ involves the Fourier transform of the slowly varying function $P\left(\boldsymbol{\rho}_{1}\right)$. This function is assumed to contain only components of small spatial frequency. Similarly, the propagation from $z_{3} \rightarrow z_{2}$ requires only the Fourier transform of the slowly varying function $Q\left(\rho_{3}\right)$, rather than of the complete rapidly oscillating wavefield $\psi\left(\boldsymbol{\rho}_{3}, z_{3}\right)$. The function $Q\left(\boldsymbol{\rho}_{3}\right)$ is constrained to have an amplitude deduced from the intensity data in the detector plane, so that its phase is determined iteratively. The determination of this phase distribution simultaneously fixes the wavefields in the planes at $z_{1}, z_{2}$ and $z_{3}$. Any other wavefield in a plane in the interval $z_{1} \leq z_{t} \leq z_{3}$ may, as a consequence, be determined by direct propagation from any of these known planar fields using equation (1). For the purposes of coherent diffractive imaging, $z_{t}$ is defined by the position of any sample placed within the beam.

The phase retrieval algorithm may be summarized by the following steps, derived from specific cases of equation (1) and the substitutions defined by equations (2) and (3).

(1) Propagate $z_{1} \rightarrow z_{2} ; \psi\left(\boldsymbol{\rho}_{2}, z_{2}\right)=A\left(\boldsymbol{\rho}_{2}, z_{12}\right) \hat{F}\left[P\left(\boldsymbol{\rho}_{1}\right)\right]$.

(2) Propagate $z_{2} \rightarrow z_{3} ; Q\left(\boldsymbol{\rho}_{3}\right)=-i \exp \left(2 \pi i z_{23} / \lambda\right) \hat{F}\left[B\left(\boldsymbol{\rho}_{2}, z_{23}\right) \psi\left(\boldsymbol{\rho}_{2}, z_{2}\right)\right]$.

(3) Impose wavefield amplitude constraint on $Q\left(\rho_{3}\right)$.

(4) Propagate $z_{3} \rightarrow z_{2} ; \psi\left(\boldsymbol{\rho}_{2}, z_{2}\right)=A\left(\boldsymbol{\rho}_{2}, z_{32}\right) \hat{F}\left[Q\left(\boldsymbol{\rho}_{3}\right)\right]$.

(5) Propagate $z_{2} \rightarrow z_{1} ; P\left(\boldsymbol{\rho}_{1}\right)=-i \exp \left(2 \pi i z_{21} / \lambda\right) \hat{F}\left[B\left(\boldsymbol{\rho}_{2}, z_{21}\right) \psi\left(\boldsymbol{\rho}_{2}, z_{2}\right)\right]$.

(6) Impose support constraint on $P\left(\rho_{1}\right)$.

The procedure is initialized with a guessed form for $P\left(\rho_{1}\right)$. Steps $1-6$ are repeated until a self-consistent solution is obtained, determined by the mean-square error in $Q\left(\rho_{3}\right)$ at the conclusion of step 2 falling below a certain value. Although the oscillatory functions $A\left(\boldsymbol{\rho}_{j}, z_{i j}\right)$ and $B\left(\boldsymbol{\rho}_{i}, z_{i j}\right)$ appear in the algorithm, they are only ever used to multiply $\psi\left(\rho_{2}, z_{2}\right)$. The focal distribution is so highly localized in the neighbourhood of $\rho_{2}=0$ that the highly oscillatory regions of these functions make no contribution to the propagation algorithm from $z_{2}$ in either direction. Each of the four Fourier transformations appearing in the algorithm are then adequately sampled, preserving the numerical stability of the algorithm.

Received 27 October 2005; accepted 3 January 2006; published 29 January 2006.

\section{References}

1. Rundquist, A. et al. Phase-matched generation of coherent soft X-rays. Science 280, 1412-1415 (1998).

2. Brinkmann, R., Materlik, G., Rossbach, J., Schneider, J. R. \& Wiik, B. H. An X-ray Fel laboratory as part of a linear collider design. Nucl. Instrum. Methods Phys. Res. A 393, 86-92 (1997).

3. Neutze, R., Wouts, R., van der Spoel, D., Weckert, E. \& Hajdu, J. Potential for biomolecular imaging with femtosecond X-ray pulses. Nature 406, 752-757 (2000).

4. Bates, R. H. T. Fourier phase problems are uniquely solvable in more than one dimension. 1. Underlying theory. Optik 61, 247-262 (1982).

5. Fienup, J. R. Phase retrieval algorithms—a comparison. Appl. Opt. 21, 2758-2769 (1982).

6. Gerchberg, R. W. \& Saxton, W. O. Practical algorithm for determination of phase from image and diffraction plane pictures. Optik 35, 237-246 (1972)

7. Miao, J. W., Charalambous, P., Kirz, J. \& Sayre, D. Extending the methodology of X-ray crystallography to allow imaging of micrometre-sized non-crystalline specimens. Nature $\mathbf{4 0 0}$, 342-344 (1999).

8. Marchesini, S. et al. X-ray image reconstruction from a diffraction pattern alone. Phys. Rev. B 68, 140101 (2003).

9. Miao, J. W., Sayre, D. \& Chapman, H. N. Phase retrieval from the magnitude of the Fourier transforms of nonperiodic objects. J. Opt. Soc. Am. A 15, 1662-1669 (1998).

10. Fienup, J. R. Phase-retrieval algorithms for a complicated optical-system. Appl. Opt. 32 , 1737-1746 (1993)

11. Fienup, J. R., Marron, J. C., Schulz, T. J. \& Seldin, J. H. Hubble space telescope characterized by using phase-retrieval algorithms. Appl. Opt. 32, 1747-1767 (1993).

12. Chapman, H. N. Phase-retrieval X-ray microscopy by Wigner-distribution deconvolution. Ultramicroscopy 66, 153-172 (1996).

13. Pitts, T. A. \& Greenleaf, J. F. Fresnel transform phase retrieval from magnitude. IEEE Trans. Ultrason. Ferroelectr. Freq. Control 50, 1035-1045 (2003).

14. Quiney, H. M., Nugent, K. A. \& Peele, A. G. Iterative image reconstruction algorithms using wave-front intensity and phase variation. Opt. Lett. 30, 1638-1640 (2005).

15. Chao, W. L., Harteneck, B. D., Liddle, J. A., Anderson, E. H. \& Attwood, D. T. Soft X-ray microscopy at a spatial resolution better than $15 \mathrm{~nm}$. Nature 435, 1210-1213 (2005).

16. Lin, J. J. A. et al. Measurement of the spatial coherence function of undulator radiation using a phase mask. Phys. Rev. Lett. 90, 074801 (2003).

17. Yun, W. et al. Nanometer focusing of hard $\mathrm{x}$ rays by phase zone plates. Rev. Sci. Instrum. 70, 2238-2241 (1999).

\section{Acknowledgements}

We acknowledge C. Tran, B. Dhal and B. Lai for discussions and assistance in acquiring the experimental data. We also acknowledge support from the Australian Synchrotron Research Program and the Australian Research Council (ARC) under the Centre of Excellence, QEII and Federation Fellowship programs. The use of the Advanced Photon Source was supported by the US Department of Energy, Office of Science, Office of Basic Energy Sciences, under Contract No. W-31-109-ENG-38. Correspondence and requests for materials should be addressed to K.A.N.

\section{Competing financial interests}

The authors declare that they have no competing financial interests.

Reprints and permission information is available online at http://npg.nature.com/reprintsandpermissions/ 